\section{Quelle prise en charge psycho-oncologique pour les patients les plus âgés ?}

\section{What kind of psycho-oncological care for the older patients?}

\author{
S. Dauchy $\cdot$ B. Desclaux $\cdot$ F. Ellien \\ (C) Springer-Verlag France 2012
}

Le vieillissement dans les pays développés est l'un des plus notables acquis des progrès de la médecine et des technologies. L'incidence du cancer augmentant avec l'âge, un tiers des patients atteints de cancer est âgé aujourd'hui de plus de 75 ans. La prise en charge de ces patients âgés atteints d'un cancer est devenue un véritable enjeu de santé publique, à la base du développement de l'oncogériatrie, qui par la collaboration entre équipes gériatriques et oncologiques vise à proposer à ces patients une prise en charge adaptée à leurs spécificités.

La psycho-oncologie a un rôle à jouer dans ces prises en charge. Discipline ancrée au cœur de l'interdisciplinarité de la cancérologie, elle doit contribuer à la prise en compte des enjeux psychologiques et sociaux engagés auprès de cette population, du côté des patients mais aussi de leurs proches et des équipes soignantes. Quelle place peuvent et doivent prendre psychologues et psychiatres dans le parcours de soin de ces patients âgés, pour prendre en compte au mieux les conséquences du vieillissement en termes d'adaptation psychologique à la maladie, mais aussi d'information, de consentement ? Quels soins psychiques proposer, aux patients comme aux aidants qui vont porter avec eux le poids de la maladie ? Comment en faciliter l'accès chez cette population spécifique? L'évaluation psychologique va également

\footnotetext{
S. Dauchy $(\bowtie)$

IGR, 39, rue Camille-des-Moulins, F-94805 Villejuif, France e-mail : Sarah.dauchy@igr.fr

B. Desclaux

Institut Claudius-Regaud, 20-24, rue du Pont-Saint-Pierre, F-31052 Toulouse, France

F. Ellien

Réseau SPES, ZA, rue de la Bigotte, F-91750 Champcueil, France
}

permettre chez ces patients fragiles, chez lesquels se cumulent les vulnérabilités - somatiques, psychologiques, socioéconomiques - de contribuer à évaluer le rapport bénéfice/ risque des traitements proposés, de préserver au mieux leur qualité de vie.

Il faudra pour cela définir comment intégrer, dans l'organisation des soins à ces patients âgés atteints de cancer, les professionnels de la psycho-oncologie ; et que ceux-ci, psychologues et psychiatres, soient au mieux prêts à prendre en compte leurs spécificités pour leur proposer une prise en charge adaptée. Il faudra aussi favoriser les liens entre ces professionnels et les différentes disciplines qui collaborent autour de ces patients, et en particulier les équipes de gériatrie et d'oncogériatrie.

C'est la raison pour laquelle la Société Française de Psycho-Oncologie a choisi de consacrer son $28^{\mathrm{e}}$ congrès, qui s'est tenu à Toulouse du 16 au 18 novembre 2011, à la prise en charge psycho-oncologique de ces patients. Ce congrès a réuni près de 300 experts et acteurs de la psycho-oncologie, de la gérontologie, de l'oncogériatrie mais aussi des soins de support ou des soins palliatifs, des professionnels de santé mentale, des médecins, des soignants. Pour que les travaux de ce congrès et les partages d'expérience qu'il a permis contribuent au mieux à structurer un corpus de connaissances lisible pour l'ensemble des acteurs de la cancérologie, ainsi que pour les autorités de tutelle, des recommandations ont été rédigées, qui résument les dix points majeurs abordés. Bien sûr non exhaustives et synthétiques, ces recommandations ont pour but d'aider les professionnels, mais aussi les tutelles, à mieux se représenter le rôle de la psycho-oncologie dans le parcours de soins en oncogériatrie. Elles sont disponibles dans ce numéro, et sont également en ligne sur le site de la SFPO (www.sfpo.fr). Nous espérons qu'elles pourront contribuer à améliorer la prise en compte de ces enjeux et de ces spécificités du grand âge. 